\title{
Lobular pattern of choriocapillaris in pre-eclampsia with aldosteronism
}

Department of Ophthalmology, Osaka University Medical School, Japan Y Saito

\section{Department of} Ophthalmology, Kansai Rosai Hospital, Osaka, Japan

T Omoto

Department of Ophthalmology, Osaka Teishin Hospital, Japan M Fukuda

Correspondence to: Yoshihiro Saito, MD, University Medical School, 11-50 Fukushima, Fukushimaku, Osaka 553, Japan. 23 May 1990 Department of Ophthalmology, Osaka Accepted for publication

\begin{abstract}
We report a case of geographic or mosaic pattern yellowish opaque foci in the left eye of a 36-year-old woman who suffered from severe pre-eclampsia. Though the geographic lesions resulted in chorioretinal atrophy, the mosaic lesion led to no significant atrophy. These two findings were both diagnosed as manifestations of choroidal ischaemia, the former due to choroidal artery occlusion, the latter to transient insufficiency of choroidal circulation, which reflects the lobular pattern of the choriocapillaris.
\end{abstract}

Patients with pre-eclampsia sometimes suffer from ocular complications, which are believed to arise mainly from retinal or choroidal vascular disorder. As to choroidal ischaemia in toxaemia of pregnancy,,$^{12}$ serous retinal detachment and deep retinal yellowish opaque focus (YOF) are the most important findings. The present report is of a case of an uncommon manifestation of choroidal vascular disorder.

\section{Case report}

A 36-year-old woman (2 gravida, 2 para) complained of decreased vision in her left eye two days after pre-eclampsia necessitated caesarean section at 34 weeks of pregnancy. Her blood pressure was $200 / 110 \mathrm{mmHg}$, 24-hour urine protein $1750 \mathrm{mg} / \mathrm{dl}(10.4 \mathrm{mmol} / 24 \mathrm{~h})$, with generalised oedema. The red blood cell coun was $3200 \times 10^{\%} / 1$, white blood cell count $14 \cdot 9 \times$

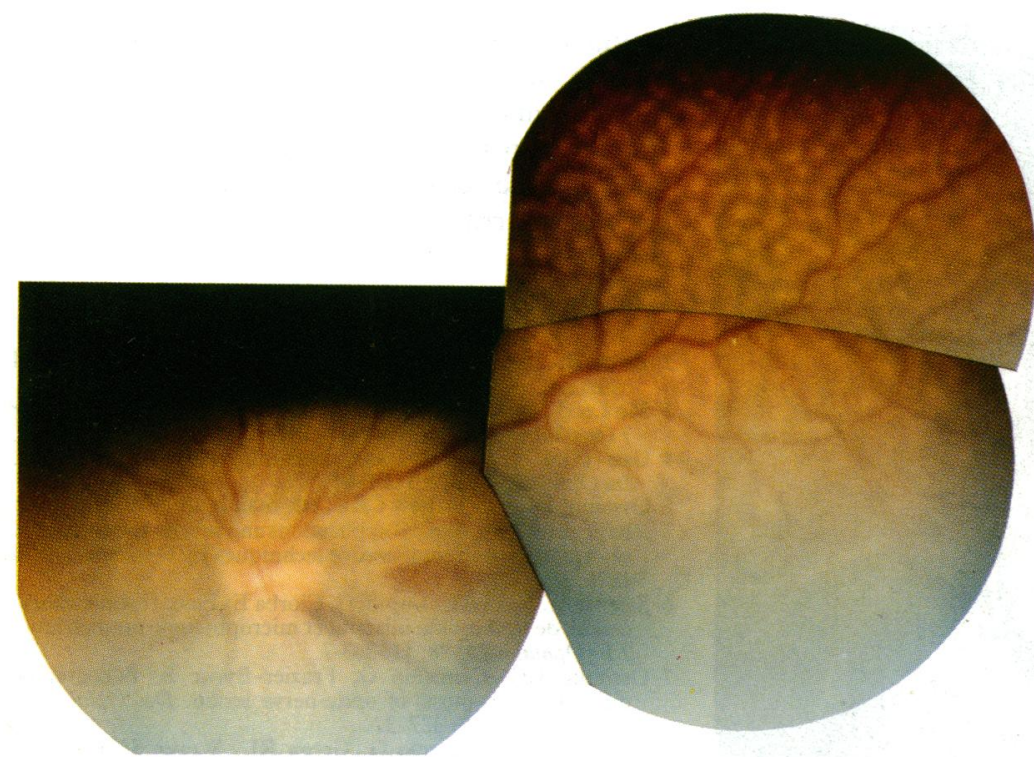

Figure 1 Left fundus at first ophthalmoscopic examination. Note peripapillary geographic yellowish opaque focus (YOF), mosaic YOF in superotemporal area, and diffuse serous retinal detachment.

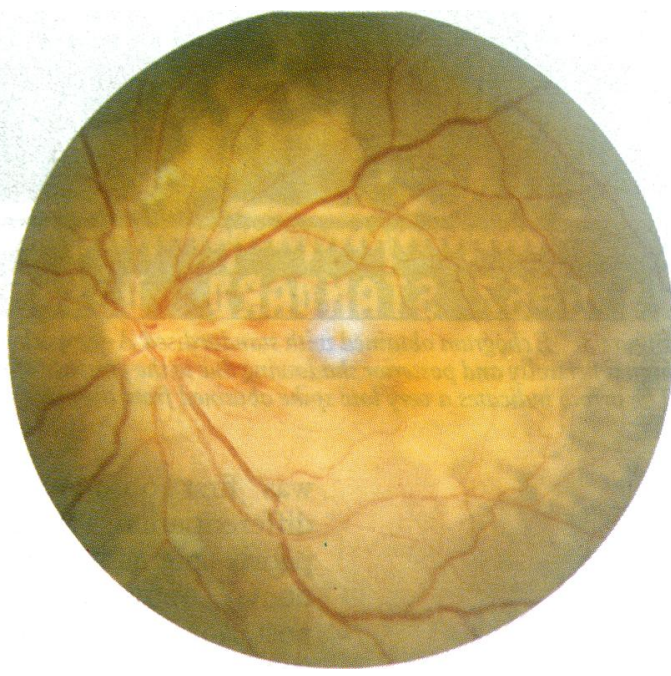

Figure 2 One week later, mosaic YOFs and retinal detachment have disappeared. Note peripapillary and temporal geographic YOFs. Central blue ring is an artefact.

$10^{9} / 1$ and platelet count $105 \times 10^{9} / 1$. The prothrombin time was $120 \%$ of normal, partial thromboplastin time 34 seconds, fibrinogen $4 \cdot 3$ $\mathrm{g} / \mathrm{l}$ and fibrin degradative product $10 \mu \mathrm{g} / \mathrm{l}$. Serum potassium was $2.8 \mathrm{mmol} / \mathrm{l}$.

At the first ophthalmological examination five days after delivery both fundi showed retinal angiospasm, flame-shaped retinal haemorrhages, cotton-wool patches, and papilloedema. In addition there were peripapillary and temporal macular geographic YOFs in the left posterior fundus at the level of the retinal pigment epithelium. Multiple isolated and some fused $1 / 4$ disc diameter YOFs were also distributed in mosaic

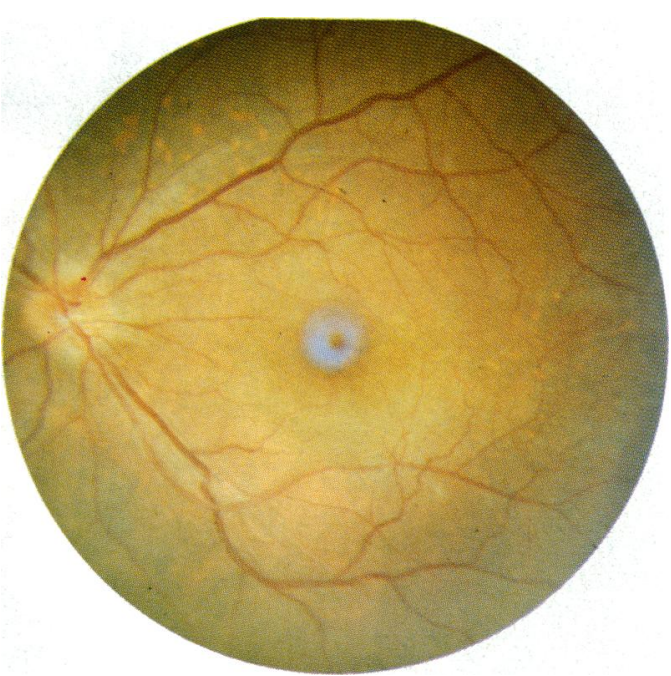

Figure 3 Three months later, chorioretinal atrophy appears at sites of previous geographic YOFs. Central blue ring is an artefact. 
fashion throughout the superotemporal to midperipheral area (Fig 1). Furthermore, diffuse serous retinal detachment involved the macula. The visual acuity of the eye was finger counting at $1 \mathrm{~m}$.

One week later, though the peripapillary and temporal geographic YOF remained, the mosaic YOFs and serous retinal detachment had disappeared (Fig 2). Fluorescein angiography was not performed at this time because of the patient's poor systemic condition.

Three months later chorioretinal atrophy was seen at the sites of the geographic YOFs but no scarring was visible ophthalmoscopically or angiographically at the sites of the mosaic YOFs (Fig 3). Visual acuity improved to 20/20. Computed tomography post partum revealed a left adrenal tumour. Partial adrenalectomy was performed. The diagnosis was primary aldosteronism due to a functional adenoma.

\section{Discussion}

In this case the geographic YOFs were thought to be caused by occlusion of a choroidal artery, as in the triangular syndrome. In addition the area of mosaic YOFs coincides with one of the frequent sites of choroidal atrophy in the tri- angular syndrome. ${ }^{3}$ Since the unit size of the mosaic YOF is the same as that of the choriocapillaris, ${ }^{4}$ these units are thought to be choroidal lobules. In the mosaic area units tend to fuse with each other, a condition presumed to be a precursor of geographic YOFs. Mosaic YOFs, which in the present case did not develop into choroidal atrophy, is also regarded as one of the manifestations of choroidal ischaemia. Presumably this mosaic YOF was not caused by a thrombosis within the choriocapillaris or by persistent occlusion of the choroidal artery. The authors speculate that such a pattern of YOFs seems to be caused by transient occlusion or spasm of the proximal choroidal artery. The presence of these two types of outcome of choroidal ischaemia in one eye provides clinical evidence of the segmental nature of the choroidal vasculature. ${ }^{5}$

1 Fastenberg DM, Fetkenhour CL, Choromokos E, et al. Choroidal vascular changes in toxemia of pregnancy. $A m \dot{\mathcal{J}}$ Ophthalmol 1980; 89: 362-8.

2 Saito Y. Retinochoroidal changes in toxemia of pregnancy with the relation to hypertensive retinopathy and choroidopathy. Nippon Ganka Gakkai Zasshi 1990; 94: 748-55.

3 Takeda M. Fluorescein angiographic findings in 'idiopathic' triangular syndrome. Folia Ophthalmol fpn 1978; 29: 1629-
to 38.

4 Hayreh SS. The choriocapillaris. Graefes Arch Clin Exp Ophthalmol 1974; 192: 165-79.

5 Hayreh SS. Segmental nature of choroidal vasculature. $\operatorname{Br} \mathcal{J}$ Ophthalmol 1975; 59: 631-48. 